\title{
Induktive Definitionen und Dilatoren
}

\author{
Wilfried Buchholz \\ Mathematisches Institut der Universität München, Theresienstrasse 39, D-8000 München 2, \\ Bundesrepublik Deutschland
}

\section{Inductive Definitions and Dilators}

Summary. In this paper we give a new and comparatively simple proof of the following theorem by Girard [1]:

"If $\forall x \in \mathcal{O} \exists y \in \mathcal{O} \psi(x, y)$ (where the relation $\psi$ is arithmetic and positive in Kleene's $(\mathcal{O})$, then there exists a recursive Dilator $D$ such that $\forall \alpha \geqq \omega \forall x \in \mathscr{O}^{<\alpha} \exists y \in \mathcal{O}^{<D(\alpha)} \psi(x, y)$."

The essential feature of our proof is its very direct definition of the dilator $D$. Within a certain infinitary cutfree system of "inductive logic" (which in fact is a modification of Girard's system in [1]) we construct in a uniform way for each ordinal $\alpha$ a derivation $T_{\alpha}$ of the formula $\forall x \in \mathcal{O}^{<\alpha} \exists y \in \mathcal{O} \psi(x, y)$, and then define $D$ immediately from the family $\left(T_{\alpha}\right)_{\alpha \in 0 n}$. Especially we set $D(\alpha):=$ KleeneBrouwer length of $T_{a}$.

In der vorliegenden Arbeit geben wir einen anderen Beweis für den folgenden Satz von Girard [1, Theorem 3.2(i), (iv)].

Satz I (Girard 1979). Gilt $\Sigma \models_{I} \varphi$ (und ist $\Sigma$ rekursiv), so gibt es einen (rekursiven) Dilator $D$ derart, daß $\Sigma \models_{I} \varphi^{\alpha, D(\alpha)}$ für alle $\alpha \in \mathrm{On}$.

Hier bezeichnet $\Sigma$ ein abzählbares Axiomensystem 1. Stufe und $\varphi$ eine Formel, in der außer den Symbolen von $\Sigma$ noch Konstanten $P_{\mathfrak{Q}}$ für induktiv definierte Teilmengen des Grundbereichs vorkommen dürfen. $\Sigma \models_{I} \varphi$ bedeutet, daß $\varphi$ in jedem Modell von $\Sigma$ gilt, welches den Konstanten $P_{\mathfrak{Q}}$ ihre Standardinterpretation zuordnet. $\varphi^{\alpha, \beta}$ entsteht aus $\varphi$ durch Einsetzen von $P_{\mathscr{N}}^{<\alpha}$ (bzw. $P_{\mathscr{\mathscr { O }}}^{<\beta}$ ) für jedes

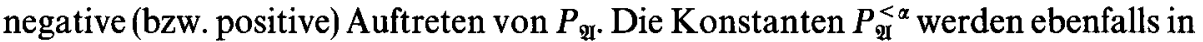
kanonischer Weise interpretiert. Ein Dilator ist ein Funktor von ON in ON, der direkte Limites und pull-backs erhält. $O N$ ist die Kategorie der Ordinalzahlen mit den streng monoton wachsenden Funktionen als Morphismen. Der obige Satz hat einige bedeutsame Anwendungen in der Rekursions- und Definierbarkeitstheorie gefunden, auf die hier aber nicht näher eingegangen werden soll (s. z. B.: $[4,5,7,8]$ ). Wir zitieren nur zwei einfache Folgerungen aus Satz I: 
Satz II (Girard 1979). Gilt $\forall x \in \mathcal{O}$ 盺 $y \in \mathcal{O} \psi(\mathcal{O}, x, y)$ (wobei $\psi$ arithmetisch und positiv in $\mathcal{O})$, so gibt es einen rekursiven Dilator $D$ mit $\forall \alpha \geqq \omega \forall x \in \mathcal{O}^{<\alpha} \exists y \in \mathcal{O}^{<D(\alpha)} \psi(\mathcal{O}, x, y)$.

( $\mathcal{O}$ bezeichnet Kleenes System der konstruktiven Ordinalzahlen.)

Satz III (van de Wiele 1981). Ist F:On $\rightarrow$ On uniform $\Sigma_{1}$-definierbar über allen zulässigen Mengen, so gibt es einen rekursiven Dilator $D$ mit $F(\alpha) \leqq D(\alpha)$ für alle $\alpha \in$ On.

Unser Beweis von Satz I zeichnet sich durch seine äußerst direkte Konstruktion des Dilators $D$ aus, die jetzt kurz skizziert werden soll. Für $\alpha \in$ On bezeichne $\varphi_{-}^{\alpha}$ die Formel, die aus $\varphi$ durch Einsetzen von $P_{\mathfrak{I}}^{<\alpha}$ für jedes negative Auftreten von $P_{\text {II }}$ entsteht. In Verallgemeinerung der Methode aus Schütte [10, Abschn. 5] wird zu jeder Formel $\varphi_{-}^{\alpha}$ ein Formelbaum (Suchbaum) $T_{\varphi \underline{\alpha}}$ definiert, für den gilt:

(i) $\Sigma \models_{I} \varphi_{-}^{\alpha} \Rightarrow T_{\varphi^{\alpha}}$ fundiert

(ii) $T_{\varphi^{\alpha}}$ fundiert $\Rightarrow \Sigma \models_{I} \varphi^{\alpha, \tilde{\alpha}}$ mit $\tilde{\alpha}:=\left\|T_{\varphi^{\alpha}}\right\|$ (Kleene-Brouwer-Länge).

(Ein fundierter Baum $T_{\varphi \underline{\alpha}}$ läßt sich als Herleitung von $\varphi_{-}^{\alpha}$ aus $\Sigma$ in einem geeigneten infinitären Kalkül der ,induktiven Logik“" auffassen.)

Gilt nun $\Sigma \models_{I} \varphi$, so auch $\Sigma \models_{I} \varphi_{-}^{\alpha}$ für alle $\alpha \in \mathrm{On}$, und mit (i), (ii) folgt daraus $\Sigma \models_{I} \varphi^{\alpha, D(\alpha)}$ für alle $\alpha \in \mathrm{On}$, wobei $D(\alpha):=\left\|T_{\varphi^{\alpha}}\right\|$. Die Funktion $D:$ On $\rightarrow$ On, $\alpha \mapsto\left\|T_{\varphi \underline{\alpha}}\right\|$ wird durch folgende Definition von $D(f) \in \operatorname{Mor}(D(\alpha), D(\beta))$ für $f \in \operatorname{Mor}(\alpha, \beta)$ zu einem Dilator: Sei $\gamma \in D(\alpha)$ und $s$ der Knoten von $T_{\varphi^{\alpha}}$, welcher in der Kleene-Brouwer-Ordnung von $T_{\varphi \underline{\alpha}}$ and $\gamma$-ter Stelle steht. Der Knoten $s$ ist eine endliche Folge von Ordinalzahlen $<\alpha$ und gewissen Symbolen $*_{0}, *_{1}$. Ersetzt man in $s$ jede Ordinalzahl $\xi$ durch $f(\xi)$, so erhält man einen Knoten $s^{\prime}$ von $T_{\varphi \underline{\beta}}$. Es sei

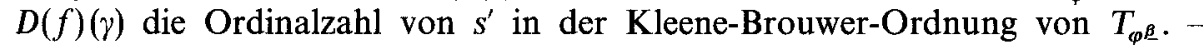
Zusammenfassend können wir feststellen, daß der Dilator $D$ unmittelbar durch die Familie der Herleitungen $T_{\varphi \underline{\alpha}}(\alpha \in \mathrm{On})$ erzeugt wird.

Bemerkung. Der Inhalt der vorliegenden Arbeit deckt sich mit dem meiner handschriftlichen Notizen „Induktive Definitionen und Dilatoren“ aus dem Jahr 1982, die inzwischen verschiedentlich zitiert wurden.

\section{Induktive Logik}

$L$ sei eine abzählbare Sprache 1. Stufe. Eine positive Operatorform ist eine Formel $\mathfrak{U}(X, x)$ der Sprache $L(X)$ ( $X$ eine Mengenvariable), in der höchstens die Variablen $X, x$ frei vorkommen und außerdem $X$ nur positiv auftritt. Jeder positiven Operatorform $\mathfrak{A}(X, x)$ werden Mengenkonstanten $P_{\mathfrak{M}}$ und $P_{\mathfrak{A}}^{<\alpha}(\alpha \in \mathrm{On})$ zugeordnet, die nicht zu $L$ gehören

$$
\begin{gathered}
L_{I D}:=L \cup\left\{P_{\mathfrak{M}}: \mathfrak{A} \text { positive Operatorform }\right\} \\
L^{\prime}:=L_{I D} \cup\left\{P_{\mathfrak{M}}^{<\alpha}: \mathfrak{A} \text { positive Operatorform und } \alpha \in \mathrm{On}\right\} .
\end{gathered}
$$

Als Mitteilungszeichen verwenden wir u.a.: $A, B, C, F$ für $L^{\prime}$-Formeln, $\varphi, \psi$ für $L_{I D^{-}}$ Formeln, $t, t_{i}$ für $L$-Terme, $\alpha, \beta, \gamma, \delta, \xi, \eta$ für Ordinalzahlen. $\Sigma$ bezeichne eine beliebige (im folgenden feste) Menge von geschlossenen $L$-Formeln. Unter einer $L$-Struktur $\mathfrak{M}$ verstehen wir hier stets eine $L$-Struktur im üblichen Sinn zusammen mit einer Belegung $x \mapsto x^{\mathfrak{M}} \in|\mathfrak{M}|$ der (freien) Variablen. 
Definition von $\mathfrak{M}{ }^{\prime}$. Ist $\mathfrak{M}$ eine $L$-Struktur, so sei $\mathfrak{M}^{\prime}$ die $L^{\prime}$-Expansion von $\mathfrak{M}$ mit

$$
\mathfrak{M}^{\prime}\left(P_{\mathfrak{A}}\right):=\bigcup_{\xi \in \mathrm{On}} I_{\mathfrak{Q}}^{\xi} \quad \text { und } \quad \mathfrak{M}^{\prime}\left(P_{\mathfrak{A}}^{<\alpha}\right):=\bigcup_{\xi<\alpha} I_{\mathfrak{Q}}^{\xi},
$$

wobei

$$
I_{\mathfrak{Q}}^{\xi}:=\left\{a \in|\mathfrak{M}|: \mathfrak{M}=\mathfrak{A}\left(\bigcup_{\eta<\xi} I_{\mathfrak{Q}}^{\eta}, a\right)\right\}
$$

Definition von $\Sigma \models_{I} A$

$\Sigma \models_{I} A: \Leftrightarrow$ Für jedes Modell $\mathfrak{M}$ von $\Sigma$ gilt $\mathfrak{M}^{\prime}=A$.

Das halbformale System $I D_{1}^{\infty}(\Sigma)$

Formeln werden aus Primformeln und negierten Primformeln mittels $\wedge, \vee, \forall, \exists$ gebildet. Die Negation $\neg A$ einer zusammengesetzten Formel $A$ wird gemäß den de Morganschen Regeln definiert.

Definition von $A_{+}^{\alpha}, A_{-}^{\alpha}, A^{\alpha, \beta} . A_{+}^{\alpha}$ (bzw. $\left.A_{-}^{\alpha}\right)$ entstehe aus $A$ dadurch, daß jedes positive (bzw. negative) Auftreten von $P_{\mathfrak{I}}$ durch $P_{\mathfrak{g}}^{<\alpha}$ ersetzt wird. Ferner sei $A^{\alpha, \beta}:=\left(A_{-}^{\alpha}\right)_{+}^{\beta}$.

\section{Induktive Definition der Formelklasse FOR}

1. Ist $A$ eine $L$-Primformel, so $A \in \mathrm{FOR}$ und $\neg A \in \mathrm{FOR}$.

2. Alle Formeln $t \in P_{\mathfrak{Q}}$ und $t \notin P_{\mathfrak{N}}^{<\alpha}$ gehören zu FOR.

3. Mit $A, B$ gehören auch $A \wedge B, A \vee B, \forall x A, \exists x A$ zu FOR.

$F \in$ FOR gilt also genau dann, wenn $P_{\mathfrak{U}}$ nur positiv und $P_{\mathfrak{Q}}^{<\alpha}$ nur negativ in $F$ auftritt.

Vereinbarung. $F$ bezeichne im folgenden stets eine Formel aus FOR.

Definition der Menge Pos $(C)$ der Positivteile einer Formel $C$

$$
\operatorname{Pos}(C):= \begin{cases}\{C\} \cup \operatorname{Pos}(A) \cup \operatorname{Pos}(B), & \text { falls } C=A \vee B \\ \{C\}, & \text { sonst } .\end{cases}
$$

Logische Axiome. Eine Formel $C$ ist ein (logisches) Axiom, wenn es eine $L$-Primformel $A$ gibt, so daß $A \in \operatorname{Pos}(C)$ und $\neg A \in \operatorname{Pos}(C)$.

Schlußregeln für $P_{\mathfrak{U}}$ und $P_{\mathfrak{U}}^{<\alpha}$

$$
\begin{array}{ll}
\left(P_{\mathfrak{I}}\right) & \mathfrak{U}\left(P_{\mathfrak{Q}}, t\right) \vee C \vdash t \in P_{\mathfrak{I}} \vee C \\
\left(P_{\mathfrak{U}}^{<\alpha}\right) & \ldots \neg \mathfrak{U}\left(P_{\mathfrak{Q}}^{<\xi}, t\right) \vee C \ldots(\xi<\alpha) \vdash t \notin P_{\mathfrak{A}}^{<\alpha} \vee C .
\end{array}
$$

Spezielle Schnittregel

( $\Sigma) \quad G \vee C \vdash C$, falls $G \in \tilde{\Sigma}$.

Dabei sei

$$
\widetilde{\Sigma}:=\Sigma \cup\{\forall x(x=x)\} \cup\{\forall x \forall y(x \neq y \vee \neg B(x) \vee B(y)): B \text { L-Primformel }\} .
$$


Das halbformale System $I D_{1}^{\infty}(\Sigma)$ bestehe aus den oben genannten logischen Axiomen, den Schlußregeln $\left(P_{\mathfrak{Y}}\right),\left(P_{\mathfrak{g}}^{<\alpha}\right),(\Sigma)$ und den üblichen Schlußregeln für $\wedge$, $\vee, \forall, \exists$. In Abschn. 2 werden wir beweisen, daß dieses System vollständig für die Formeln aus FOR ist, d.h. daß jede Formel der Klasse $\left\{F \in \mathrm{FOR}: \Sigma \models_{I} F\right\}$ in $I D_{1}^{\infty}(\Sigma)$ hergeleitet werden kann.

Vorher soll noch gezeigt werden, wie Satz II aus Satz I folgt: Sei $L:=\{0,1,+, \cdot\}$ und $\Sigma:=P A$ (Peano-Arithmetik),

$$
\begin{gathered}
\mathfrak{A}_{0}(X, x):=(x=0) \vee \exists y(y \in X \wedge x=y+1), \\
\mathfrak{A}_{1}(X, x):=(x=0) \vee \exists y\left(y \in X \wedge x=2^{y}\right) \vee \exists y\left(x=3 \cdot 5^{y} \wedge \forall z\{y\}(z) \in X\right), \\
P_{i}:=P_{\mathfrak{A}_{i}}, \quad \varphi:=\forall x\left(x \in P_{0}\right) \rightarrow \forall x \in P_{1} \exists y \in P_{1} \psi\left(P_{1}, x, y\right) .
\end{gathered}
$$

Angenommen $\mathfrak{M}^{\prime} \models P A \cup\left\{\forall x\left(x \in P_{0}\right)\right\}$. Dann ist $\mathfrak{M}$ isomorph zur Standardstruktur $\mathfrak{N}$ der natürlichen Zahlen. Außerdem ist $\mathcal{O}=I_{\mathfrak{A}_{1}}$ (in $\mathfrak{N}$ ). Mit der Voraussetzung $\forall x \in \mathcal{O}$ 马ly $\in \mathcal{O} \psi(\mathcal{O}, x, y)$ folgt also $\mathfrak{M}^{\prime} \models \forall x \in P_{1} \exists y \in P_{1} \psi\left(P_{1}, x, y\right)$. Somit haben wir gezeigt: $P A \models_{I} \varphi$. Nach Satz I gibt es also einen rekursiven Dilator $D$ mit $P A \models{ }_{I} \forall x\left(x \in P_{0}^{<\alpha}\right) \rightarrow \forall x \in P_{1}^{<\alpha} \exists y \in P_{1}^{<D(\alpha)} \psi\left(P_{1}^{<D(\alpha)}, x, y\right)$, für alle $\alpha \in \mathrm{On}$. Wegen $\mathfrak{N}^{\prime}=\forall x\left(x \in P_{0}^{<\alpha}\right)$ für $\alpha \geqq \omega$, und da $P_{1}$ in $\psi$ nur positiv auftritt, folgt daraus $\mathfrak{N}^{\prime} \models \forall x \in P_{1}^{<\alpha} \exists y \in P_{1}^{<D(\alpha)} \psi\left(P_{1}, x, y\right)$, d.h. $\forall x \in \mathcal{O}^{<\alpha} \exists y \in \mathcal{O}^{<D(\alpha)} \psi(\mathcal{O}, x, y)$, für $\alpha \geqq \omega$.

\section{Die Formelbäume $T_{F}$}

\section{Induktive Definition der Positivformen}

$(\Gamma 1) *$ ist eine Positivform.

( $\Gamma 2)$ Ist $A$ eine Formel und $\Gamma$ eine Positivform, so ist auch $A \vee \Gamma$ eine Positivform. Im folgenden bezeichnen $\Gamma, \Gamma_{i}$ stets Positivformen.

Definition von $\Gamma(B)$ und $B \vee \Gamma()$

1. Für $\Gamma=*$ sei $\Gamma(B):=B \vee \Gamma():=B$.

2. Für $\Gamma=A \vee \Gamma_{0}$ sei $\Gamma(B):=A \vee \Gamma_{0}(B), B \vee \Gamma():=B \vee\left(A \vee \Gamma_{0}()\right)$.

Bemerkung. Jede Formel $C$ läßt sich auf genau eine Weise in der Form $C=\Gamma(B)$ mit $B \neq B_{0} \vee B_{1}$ darstellen.

Definition. $*_{0}, *_{1}$ seien zwei Objekte (Symbole) mit On $\cap\left\{*_{0}, *_{1}\right\}=\emptyset$. $\alpha$-SEQ $:=\left\{\left(x_{0}, \ldots, x_{n-1}\right): n \geqq 0, x_{0}, \ldots, x_{n-1} \in \alpha \cup\left\{*_{0}, *_{1}\right\}\right\}$.

$\mathrm{SEQ}:=\bigcup\{\alpha-\mathrm{SEQ}: \alpha \in \mathrm{On}\}$.

$F$ sei eine für den Rest dieses Abschnitts feste Formel aus FOR. Wir werden jetzt einen bzgl. der Schlußregeln von $I D_{1}^{\infty}(\Sigma)$ lokal korrekten Formalbaum $T_{F}$ definieren und anschließend beweisen, daß $T_{F}$ genau dann fundiert ist, wenn $\Sigma \models_{I} F$ gilt. $T_{F}$ wird als Funktion von SEQ in FOR $\cup\{\infty\}(\infty$ für „undefiniert") erklärt; diese sehr formale Definition von $T_{F}$ brauchen wir in Abschn. $3 \mathrm{um}$ die Homogenität der Familie $\left(T_{\varphi_{-}^{\alpha}}\right)_{\alpha \in \text { On }}$ nachzuweisen (vgl. [6]).

\section{Definition}

$G_{0}, G_{1}, \ldots$ sei eine Abzählung von $\tilde{\Sigma}$.

$t_{0}, t_{1}, \ldots$ sei eine Abzählung aller $L$-Terme. 
Definition des Formelbaums $T_{F}: \mathrm{SEQ} \rightarrow \mathrm{FOR} \cup\{\infty\}$

Die Definition von $T_{F}(s)$ erfolgt durch Induktion nach der Länge von $s \in \mathrm{SEQ}$.

(T1) $\quad T_{F}(()):=F$.

Sei nun $s=\left(x_{0}, \ldots, x_{n}\right)$ und $s_{0}:=\left(x_{0}, \ldots, x_{n-1}\right)$.

(T2) $T_{F}\left(s_{0}\right) \in$ FOR kein logisches Axiom, $n=2 m, x_{n}=*_{0}$ :

$T_{F}(s):=\neg G_{m} \vee T_{F}\left(s_{0}\right)$.

(T3) $T_{F}\left(s_{0}\right)=: C \in$ FOR kein logisches Axiom und $n$ ungerade:

(T3.1) $C=\Gamma\left(A_{0} \wedge A_{1}\right)$ und $x_{n}=*_{i}: T_{F}(s):=\Gamma\left(A_{i}\right)$.

(T3.2) $C=\Gamma(\forall x A(x))$ und $x_{n}=*_{0}: T_{F}(s):=\Gamma(A(v))$, dabei sei $v$ die erste nicht in $C$ vorkommende Variable.

(T3.3) $C=\Gamma(\exists x A(x))$ und $x_{n}=*_{0}: T_{F}(s):=\exists x A(x) \vee \Gamma\left(A\left(t_{k}\right)\right)$; enthält $A(x)$ wenigstens ein freies Auftreten von $x$, so sei $k$ die kleinste Zahl derart, daß $A\left(t_{k}\right)$ nicht Positivteil einer der Formeln $T_{F}\left(\left(x_{0}, \ldots, x_{i-1}\right)\right)(i=0, \ldots, n)$ ist; anderenfalls sei $k=0$.

(T3.4) $C=\Gamma\left(t \in P_{\mathfrak{Q}}\right)$ und $x_{n}=*_{0}: T_{F}(s):=\mathfrak{Q}\left(P_{\mathfrak{Q}}, t\right) \vee \Gamma()$.

(T3.5) $C=\Gamma\left(t \notin P_{\mathfrak{A}}^{<\alpha}\right)$ und $x_{n}=\xi<\alpha: T_{F}(s):=\neg \mathfrak{A}\left(P_{\mathfrak{A}}^{<\xi}, t\right) \vee \Gamma()$.

(T3.6) $C=\Gamma(A)$ und $x_{n}=*_{0}$, wobei $A$ eine (negierte) $L$-Primformel: $T_{F}(s):=A \vee \Gamma()$.

(T4) In allen anderen Fällen sei $T_{F}(s):=\infty$.

Definition. $\operatorname{dom}\left(T_{F}\right):=\left\{s \in \mathrm{SEQ}: T_{F}(s) \in \mathrm{FOR}\right\}$.

$T_{F}$ heißt fundiert, wenn es keine unendliche Folge $\left(x_{n}\right)_{n \in \mathbb{N}}$ in $O n \cup\left\{*_{0}, *_{1}\right\}$ gibt, so daß $\left(x_{0}, \ldots, x_{n-1}\right) \in \operatorname{dom}\left(T_{F}\right)$ für alle $n \in \mathbb{N}$.

Satz 1. $T_{F}$ nicht fundiert $\Rightarrow \Sigma \not{ }_{I} F$.

Beweis. Nach Voraussetzung existiert eine Folge $\left(x_{n}\right)_{n \in \mathbb{N}}$ in $O n \cup\left\{*_{0}, *_{1}\right\}$ mit $C_{n}:=T_{F}\left(\left(x_{0}, \ldots, x_{n-1}\right)\right) \in$ FOR für alle $n \in \mathbb{N}$.

Nach Definition von $T_{F}$ ist keine der Formeln $C_{n}$ ein logisches Axiom, und es gilt $C_{2 m+1}=\neg G_{m} \vee C_{2 m}$ für alle $m \in \mathbb{N}$. Aus letzterem folgt $\{\neg A: A \in \tilde{\Sigma}\} \subseteq H$.

Dabei sei $H:=\bigcup_{n \in \mathbb{N}} \operatorname{Pos}\left(C_{n}\right)$.

Hilfssatz 1. Ist $A \in \operatorname{Pos}\left(C_{n}\right)$ keine $\vee-F o r m e l$, so gibt es ein ungerades $k \geqq n$ und eine Positivform $\Gamma$ mit $C_{k}=\Gamma(A)$.

Beweis. Wir können annehmen, daß $n$ ungerade ist. Ferner beziehen wir uns auf ein bestimmtes Auftreten von $A$ in $C_{n}$ und nehmen an, $C_{n}$ sei nicht von der Gestalt $\Gamma(A)$. Nach Definition von $T_{F}$ ist dann auch $A \in \operatorname{Pos}\left(C_{n+2}\right)$, und in $C_{n+2}$ stehen rechts von $A$ weniger logische Zeichen als in $C_{n}$. So kommt man schließlich zu einer Formel $C_{n+2 i}$, in der rechts von $A$ keine logischen Zeichen stehen. Dann ist $C_{n+2 i}=\Gamma(A)$.

\section{Hilfssatz 2.}

a) $A$ Primformel von $L \Rightarrow A \notin H$ oder $\neg A \notin H$.

b) $A_{0} \wedge A_{1} \in H \Rightarrow A_{0} \in H$ oder $A_{1} \in H$.

c) $A_{0} \vee A_{1} \in H \Rightarrow A_{0} \in H$ und $A_{1} \in H$.

d) $\forall x A(x) \in H \Rightarrow$ Es gibt einen L-Term $t$ mit $A(t) \in H$.

e) $\exists x A(x) \in H \Rightarrow$ Für jeden L-Term $t$ gilt $A(t) \in H$.

f) $\left(t \notin P_{\mathfrak{q}}^{<\alpha}\right) \in H \Rightarrow$ Es gibt ein $\xi<\alpha$ mit $\neg \mathfrak{U}\left(P_{\mathfrak{U}}^{<\xi}, t\right) \in H$. 
g) $\left(t \in P_{\mathfrak{V}}\right) \in H \Rightarrow \mathfrak{Q}\left(P_{\mathfrak{V}}, t\right) \in H$.

h) Für alle L-Terme $t, t^{\prime}$ und alle L-Primformeln $B(x)$ gilt:

(i) $(t \neq t) \in H$.

(ii) $\left(t \neq t^{\prime}\right) \in H \quad$ und $\neg B(t) \in H \Rightarrow \neg B\left(t^{\prime}\right) \in H$.

Beweis. a) Sei $A$ L-Primformel. Dann gilt:

(1) $B \in\{A, \neg A\} \cap \operatorname{Pos}\left(C_{n}\right) \Rightarrow B \in \operatorname{Pos}\left(C_{k}\right)$ für alle $k \geqq n$.

Da die Folge $\left(C_{k}\right)_{k \in \mathbb{N}}$ kein logisches Axiom enthält, gilt außerdem $A \notin \operatorname{Pos}\left(C_{k}\right)$ oder $\neg A \notin \operatorname{Pos}\left(C_{k}\right)$, für alle $k \in \mathbb{N}$. Mit (1) folgt daraus die Behauptung.

b) Ist $A_{0} \wedge A_{1} \in \operatorname{Pos}\left(C_{n}\right)$, so gibt es nach Hilfssatz 1 ein ungerades $k$ mit $C_{k}=\Gamma\left(A_{0} \wedge A_{1}\right)$; folglich $C_{k+1}=\Gamma\left(A_{0}\right)$ oder $\Gamma\left(A_{1}\right)$, und somit $A_{0} \in H$ oder $A_{1} \in H$.

c) Ist trivial. d), $\mathrm{f}$, g) werden ebenso wie b) bewiesen.

e) Sei $\exists x A(x) \in H$. Dann existiert ein $k_{0}$ mit $\exists x A(x) \in \operatorname{Pos}\left(C_{n}\right)$ für alle $n \geqq k_{0}(*)$. Durch Induktion nach $k$ zeigen wir $A\left(t_{k}\right) \in H$ : Gelte $A\left(t_{i}\right) \in H$ für alle $i<k$. Wegen Hilfssatz 1 und (*) gibt es ein ungerades $n \geqq k_{0}$ mit $C_{n}=\Gamma(\exists x A(x))$ und $\left\{A\left(t_{i}\right): i<k\right\} \subseteq \bigcup_{j \leqq n} \operatorname{Pos}\left(C_{j}\right)$. Dann $\quad C_{n+1}=\exists x A(x) \vee \Gamma\left(A\left(t_{l}\right)\right) \quad$ mit $k \leqq l$ und $\left\{A\left(t_{i}\right): i<l\right\} \subseteq H$. Also $A\left(t_{k}\right) \in H$.

h) Wegen $\{\neg A: A \in \tilde{\Sigma}\} \subseteq H$ (s. oben) haben wir $\exists x(x \neq x) \in H$ und $\exists x \exists y(x=y \wedge B(x) \wedge \neg B(y)) \in H$. Mit e) folgt daraus $t \neq t \in H$ und $t=t^{\prime} \wedge B(t) \wedge \neg B\left(t^{\prime}\right) \in H$. Aus letzterem folgt mit b): $t=t^{\prime} \in H$ oder $B(t) \in H$ oder $\neg B\left(t^{\prime}\right) \in H$. Mit a) folgt daraus Behauptung (ii).

Definition. $L$-Ter : = Menge aller $L$-Terme,

$$
t \sim t^{\prime}: \Leftrightarrow t \neq t^{\prime} \in H, \quad \bar{t}:=\left\{t^{\prime} \in L-\operatorname{Ter}: t \sim t^{\prime}\right\} .
$$

Aus Hilfssatz 2h) folgt:

(i) $\sim$ ist eine Äquivalenzrelation.

(ii) $t_{1} \sim t_{1}^{\prime}, \ldots, t_{n} \sim t_{n}^{\prime} \Rightarrow f t_{1} \ldots t_{n} \sim f t_{1}^{\prime} \ldots t_{n}^{\prime}(f \in L)$.

(iii) $t_{1} \sim t_{1}^{\prime}, \ldots, t_{n} \sim t_{n}^{\prime}$ und $\neg p t_{1} \ldots t_{n} \in H \Rightarrow \neg p t_{1}^{\prime} \ldots t_{n}^{\prime} \in H(p \in L)$.

Demnach können wir eine L-Struktur $\mathfrak{M}$ wie folgt definieren:

$$
\begin{aligned}
|\mathfrak{M}| & :=\{\bar{t}: t \in L-\operatorname{Ter}\}, \quad f^{\mathfrak{M}}\left(\bar{t}_{1}, \ldots, \bar{t}_{n}\right):=\overline{f t_{1} \ldots t_{n}}, \\
x^{\mathfrak{M}}: & =\bar{x}, \quad\left(\bar{t}_{1}, \ldots, \overline{t_{n}}\right) \in p^{\mathfrak{M}}: \Leftrightarrow \neg p t_{1} \ldots t_{n} \in H .
\end{aligned}
$$

Definition von $\operatorname{gr}(A) \in \mathrm{On}$ für jede $L$-Formel $A$, in der keine Konstante $P_{\mathfrak{r}}$ vorkommt:

1. $\operatorname{gr}(A):=\operatorname{gr}(\neg A):=0$, wenn $A$ eine $L$-Primformel ist.

2. $\operatorname{gr}\left(A_{0} \wedge A_{1}\right):=\operatorname{gr}\left(A_{0} \vee A_{1}\right):=\operatorname{gr}\left(A_{0}\right) \# \operatorname{gr}\left(A_{1}\right) \# 1$.

3. $\operatorname{gr}(\forall x B):=\operatorname{gr}(\exists x B):=\operatorname{gr}(B)+1$.

4. $\operatorname{gr}\left(t \in P_{\mathfrak{M}}^{<\alpha}\right):=\operatorname{gr}\left(t \notin P_{\mathfrak{q}}^{<\alpha}\right):=\omega^{\alpha}$.

Folgerung. Für $\alpha<\beta$ gilt: $\operatorname{gr}\left(\mathfrak{A}\left(P_{\mathscr{N}}^{<\alpha}, t\right)\right)=\operatorname{gr}\left(\neg \mathfrak{A}\left(P_{\mathscr{\mathscr { A }}}^{<\alpha}, t\right)\right)<\omega^{\beta}$.

Hilfssatz 3. $C \in H \Rightarrow \mathfrak{M}^{\prime} \not \neq C_{+}^{\beta}$, für alle $\beta \in \mathrm{On}$.

Beweis. Durch Induktion nach $\operatorname{gr}\left(C_{+}^{\beta}\right)$ mittels Hilfssatz 2: Wir behandeln nur zwei Fälle. 
1. $C=t \in P_{\mathfrak{2}}$ : Dann $\mathfrak{A}\left(P_{\mathfrak{Q}}, t\right) \in H$ und nach Induktionsvoraussetzung gilt $\mathfrak{M}^{\prime} \not \neq \mathfrak{U}\left(P_{\mathscr{\ell}}^{<\xi}, t\right)$ für alle $\xi<\beta$. Folglich $\mathfrak{M}^{\prime} \not \forall t \in P_{\mathfrak{U}}^{<\beta}$.

2. $C=t \notin P_{\mathscr{\vartheta}}^{<\alpha}$ : Dann $\neg \mathfrak{A}\left(P_{\mathscr{\Re}}^{<\xi}, t\right) \in H$ für ein $\xi<\alpha$. Mit der Induktionsvoraussetzung folgt daraus $\mathfrak{M}^{\prime}=\mathfrak{H}\left(P_{\mathscr{\mathscr { N }}}^{<\xi}, t\right)$ und weiter $\mathfrak{M}^{\prime} \models t \in P_{\mathfrak{A}}^{<\alpha}$, d.h. $\mathfrak{M}^{\prime} \not C_{+}^{\beta}$, denn $C_{+}^{\beta}=C$.

Die Fälle $C=t \notin P_{\mathfrak{V}}, C=t \in P_{\mathfrak{\mathscr { H }}}^{<\alpha}$ kommen nicht vor, da $F \in \mathrm{FOR}$.

Hilfssatz 4. $\mathfrak{M} \models \Sigma$ und $\mathfrak{M}^{\prime} \not \neq F$, also $\Sigma \not{ }_{I} F$.

Beweis. 1. Sei $A \in \Sigma$. Dann $\neg A \in H$ und nach Hilfssatz 3 gilt $\mathfrak{M}^{\prime} \not(\neg A)_{+}^{0}$. Da $A$ ein $L$-Satz ist, gilt $(\neg A)_{+}^{0}=\neg A$ und somit $\mathfrak{M} \models A$.

2. Wegen $F=C_{0} \in H$ gilt nach Hilfssatz $3 \mathfrak{M}^{\prime} \not \neq F_{+}^{\beta}$ für alle $\beta \in \mathrm{On}$. Wir wählen $\beta \in$ On so groß, daß $I_{\mathfrak{Q}}=I_{\mathfrak{I}}^{<\beta}$ für jede in $F$ vorkommende Konstante $P_{\mathfrak{Q P}}$. Aus $\mathfrak{M}^{\prime} \mid \neq F_{+}^{\beta}$ folgt dann $\mathfrak{M}^{\prime} \not \neq F$.

Definition. Für $x, y \in \mathrm{On} \cup\left\{*_{0}, *_{1}\right\}$ sei:

$x<y: \Leftrightarrow x \in y \in$ On oder $\left(x \in\left\{*_{0}, *_{1}\right\} \& y \in \mathrm{On}\right)$ oder $\left(x=*_{0} \& y=*_{1}\right)$.

Mit $\prec$ bezeichnen wir die durch $<$ induzierte Kleene-Brouwer-Ordnung auf SEQ.

Bemerkung. Offenbar gilt $\operatorname{dom}\left(T_{F}\right) \subseteq \alpha_{0}$-SEQ mit

$$
\alpha_{0}:=\max \left\{\alpha: \alpha=0 \quad \text { oder } \quad P_{\mathfrak{H}}^{<\alpha} \text { kommt in } F \text { vor }\right\},
$$

d.h. $\operatorname{dom}\left(T_{F}\right)$ ist eine Menge.

Definition. Ist $T_{F}$ fundiert, so bezeichne $\left\|T_{F}\right\| \in$ On den Ordnungstyp der Wohlordnung $\left(\operatorname{dom}\left(T_{F}\right), \prec\right)$, und $\ell_{F}:\left\|T_{F}\right\| \rightarrow \operatorname{dom}\left(T_{F}\right)$ sei die zugehörige Ordnungsfunktion.

Satz 2. $T_{F}$ fundiert und $\left\|T_{F}\right\| \leqq \beta \Rightarrow \Sigma \models_{I} F_{+}^{\beta}$.

Korollar. $\Sigma \models_{I} F \Leftrightarrow T_{F}$ fundiert.

Beweis. Durch Induktion nach $\alpha$ wird gezeigt:

$$
\alpha \in\left\|T_{F}\right\| \& A=T_{F}\left(\ell_{F}(\alpha)\right) \& \alpha \leqq \beta \Rightarrow \Sigma \models_{I} A_{+}^{\beta} .
$$

Das Korollar folgt unmittelbar aus Satz 1, Satz 2 und $\Sigma \models_{I} F_{+}^{\beta} \rightarrow F$.

\section{Der Dilator $D$}

$\varphi$ sei eine im folgenden feste $L_{I D}$-Formel mit $\Sigma \models_{I} \varphi$. Dann gilt offenbar auch $\Sigma \models_{I} \varphi_{-}^{\alpha}$ und $\varphi_{-}^{\alpha} \in$ FOR für jedes $\alpha \in$ On. Nach Satz 1 ist deshalb $T_{\varphi \underline{\alpha}}$ fundiert und somit $\left\|T_{\varphi \underline{\alpha}}\right\| \in$ On definiert.

Abkürzung. $T_{\alpha}:=T_{\varphi \underline{\alpha}}, \ell_{\alpha}:=\ell_{\varphi \underline{\alpha}}$.

Bemerkung. 1. $\operatorname{dom}\left(T_{\alpha}\right) \subseteq \alpha$-SEQ.

2. $\ell_{\alpha}:\left\|T_{\alpha}\right\| \rightarrow \operatorname{dom}\left(T_{\alpha}\right)$ ist die Ordnungsfunktion der Wohlordnung $\left(\operatorname{dom}\left(T_{\alpha}\right), \prec\right)$.

Definitionen. 1. Für $s \in \mathrm{SEQ}$ sei

On $(s):=$ Menge der in $s$ vorkommenden Ordinalzahlen 
$I(s):=$ Klasse aller ordnungstreuen Funktionen $f: M \rightarrow$ On mit $\mathrm{On}(s) \subseteq M \subseteq$ On

2. Für $s=\left(x_{0}, \ldots, x_{n-1}\right) \in$ SEQ und $f \in I(s)$ sei

$$
\hat{f}(s):=\left(f\left(x_{0}\right), \ldots, f\left(x_{n-1}\right)\right) ; \text { wobei } f\left(*_{i}\right):=*_{i} .
$$

3. Für $\alpha, \beta \in$ On sei

$$
I(\alpha, \beta):=\text { Menge aller ordnungstreuen Funktionen } f: \alpha \rightarrow \beta \text {. }
$$

Folgerung. $s \in \alpha-\mathrm{SEQ} \Rightarrow I(\alpha, \beta) \subseteq I(s)$.

Satz 3. (Homogenität von $\left(T_{\alpha}\right)_{\alpha \in \text { Onn }}$ ).

$s \in \operatorname{dom}\left(T_{\alpha}\right), f \in I(s)$ und $\forall \xi \in \mathrm{On}(s)(f(\xi)<\beta) \Rightarrow \hat{f}(s) \in \operatorname{dom}\left(T_{\beta}\right)$.

Beweis folgt unten.

\section{Definition von $D: \mathrm{ON} \rightarrow \mathrm{ON}$}

(D1) $D(\alpha):=\left\|T_{\alpha}\right\| \quad(\alpha \in \mathrm{On})$

(D2) Für $f \in I(\alpha, \beta)$ sei $D(f):=\ell_{\beta}^{-1} \circ \hat{f}_{\circ} \ell_{\alpha}: D(\alpha) \rightarrow D(\beta)$. (Wegen Satz 3 ist $\hat{f} \circ \ell_{\alpha}[D(\alpha)] \subseteq \operatorname{dom}\left(T_{\beta}\right)$ und somit $\ell_{\beta}^{-1} \circ \hat{f}_{0} \circ \ell_{\alpha}(\gamma)$ für jedes $\gamma \in D(\alpha)$ definiert.)

Da $T_{\alpha}=T_{\varphi \underline{\alpha}}$ fundiert ist, gilt nach Satz $2 \Sigma \models_{I} \varphi^{\alpha, D(\alpha)}$. Zum Beweis von Satz I muß also nur noch gezeigt werden, daß das oben definierte $D$ ein (rekursiver) Dilator ist.

Definition. $\mathscr{B}:=\left\{(s, n): n \in \mathbb{N}, s \in \operatorname{dom}\left(T_{n}\right), \operatorname{On}(s)=\{0, \ldots, n-1\}\right\}$. Für $(s, n) \in \mathscr{B}$, $\alpha_{0}<\ldots<\alpha_{n-1}<\alpha$ sei $\left(s ; \alpha_{0}, \ldots, \alpha_{n-1} ; \alpha\right):=\ell_{\alpha}^{-1}(\hat{f}(s))$, wobei $f:=\left\{\left(i, \alpha_{i}\right): i<n\right\}$. [Nach Satz 3 ist $\hat{f}(s) \in \operatorname{dom}\left(T_{\alpha}\right)$.]

Lemma. a) $D(\alpha)=\left\{\left(s ; \alpha_{0}, \ldots, \alpha_{n-1} ; \alpha\right):(s, n) \in \mathscr{B}, \alpha_{0}<\ldots<\alpha_{n-1}<\alpha\right\}$.

b) Aus $\quad(s, n),\left(s^{\prime}, m\right) \in \mathscr{B}, \quad \alpha_{0}<\ldots<\alpha_{n-1}<\alpha, \quad \beta_{0}<\ldots<\beta_{m-1}<\alpha \quad$ und $\left(s ; \alpha_{0}, \ldots, \alpha_{n-1} ; \alpha\right)=\left(s^{\prime} ; \beta_{0}, \ldots, \beta_{m-1} ; \alpha\right)$ folgt $s=s^{\prime}, n=m$ und $\alpha_{i}=\beta_{i}$ für $i<n$.

c) Seien $\quad(s, n),\left(s^{\prime}, m\right) \in \mathscr{B}, \quad \alpha_{0}<\ldots<\alpha_{n-1}<\alpha, \quad \beta_{0}<\ldots<\beta_{m-1}<\alpha$, $\alpha_{0}^{\prime}<\ldots<\alpha_{n-1}^{\prime}<\alpha^{\prime}, \beta_{0}^{\prime}<\ldots<\beta_{m-1}^{\prime}<\alpha^{\prime}$, so da $\beta h:=\left\{\left(\alpha_{i}, \alpha_{i}^{\prime}\right): i<n\right\} \cup\left\{\left(\beta_{j}, \beta_{j}^{\prime}\right): j<m\right\}$ eine ordnungstreue Funktion ist. - Dann gilt:

$$
\left(s ; \alpha_{0}, \ldots, \alpha_{n-1} ; \alpha\right)<\left(s^{\prime} ; \beta_{0}, \ldots, \beta_{m-1} ; \alpha\right)
$$

impliziert

$$
\left(s ; \alpha_{0}^{\prime}, \ldots, \alpha_{n-1}^{\prime} ; \alpha^{\prime}\right)<\left(s^{\prime} ; \beta_{0}^{\prime}, \ldots, \beta_{m-1}^{\prime} ; \alpha^{\prime}\right) .
$$

d) $\operatorname{Aus}(s, n) \in \mathscr{B}, \alpha_{0}<\ldots<\alpha_{n-1}<\alpha$ und $f \in I(\alpha, \beta)$ folgt $D(f)\left(\left(s ; \alpha_{0}, \ldots, \alpha_{n-1} ; \alpha\right)\right)$ $=\left(s ; f\left(\alpha_{0}\right), \ldots, f\left(\alpha_{n-1}\right) ; \beta\right)$.

Bemerkung. Aus diesem Lemma zusammen mit Girard [3, Theorem 2.2] folgt, daß $D$ ein Dilator ist.

Beweis des Lemmas. a) Folgt unmittelbar aus Satz 3.

b) Sei $f:=\left\{\left(i, \alpha_{i}\right): i<n\right\}, g:=\left\{\left(j, \beta_{j}\right): j<m\right\}$. Dann gilt $\ell_{\alpha}^{-1} \hat{f}(s)=\ell_{\alpha}^{-1} \hat{g}\left(s^{\prime}\right)$ und folglich $\hat{f}(s)=\hat{g}\left(s^{\prime}\right)$. Daraus folgt $\left\{\alpha_{0}, \ldots, \alpha_{n-1}\right\}=\operatorname{On}(f(s))=\operatorname{On}\left(g\left(s^{\prime}\right)\right)$ $=\left\{\beta_{0}, \ldots, \beta_{m-1}\right\}$ und somit $f=g$. Aus $\hat{f}(s)=\hat{g}\left(s^{\prime}\right)$ und $f=g$ folgt $s=s^{\prime}$.

c) Sei $f:=\left\{\left(i, \alpha_{i}\right): i<n\right\}, \quad g:=\left\{\left(j, \beta_{j}\right): j<m\right\}$. Aus $\left(s ; \alpha_{0}, \ldots, \alpha_{n-1} ; \alpha\right)$ $<\left(s^{\prime} ; \beta_{0}, \ldots, \beta_{m-1} ; \alpha\right)$ folgt $\hat{f}(s)<\hat{g}\left(s^{\prime}\right)$ und weiter $\widehat{h \circ f}(s)=\hat{h} \circ \widehat{f}(s)<\hat{h} \circ \hat{g}\left(s^{\prime}\right)$ 
$=\widehat{h \circ g}\left(s^{\prime}\right) \quad$ und schließlich $\left(s ; \alpha_{0}^{\prime}, \ldots, \alpha_{n-1}^{\prime} ; \alpha^{\prime}\right)=\ell_{\alpha}^{-1} \widehat{h \circ f}(s)<\ell_{\alpha}^{-1} \widehat{h \circ g}\left(s^{\prime}\right)$ $=\left(s^{\prime} ; \beta_{0}, \ldots, \beta_{m-1} ; \alpha^{\prime}\right)$.

d) Sei $g:=\left\{\left(i, \alpha_{i}\right): i<n\right\}$.

$$
\begin{aligned}
D(f)\left(\left(s ; \alpha_{0}, \ldots, \alpha_{n-1} ; \alpha\right)\right) & =\ell_{\beta}^{-1} \widehat{f} \ell_{\alpha} \ell_{\alpha}^{-1} \hat{g}(s)=\ell_{\beta}^{-1} \widehat{f \circ g}(s) \\
& =\left(s ; f\left(\alpha_{0}\right), \ldots, f\left(\alpha_{n-1}\right) ; \beta\right) .
\end{aligned}
$$

Beweis von Satz 3. Sei $s \in \operatorname{dom}\left(T_{\alpha}\right), f \in I(s)$ und $\forall \xi \in \mathrm{On}(s)(f(\xi)<\beta)$. Dann können wir ohne Einschränkung annehmen, daß $f(\alpha)=\beta$. Für $C \in \mathrm{FOR}$ sei On $(C):=\left\{\xi \in \mathrm{On}: P_{\mathscr{U}}^{<\xi}\right.$ kommt in $C$ vor $\}$. Ist $\mathrm{On}(C) \subseteq \mathrm{On}(s) \cup\{\alpha\}$, so sei $\hat{f}(C)$ diejenige Formel, die aus $C$ entsteht, wenn jede Konstante $P_{\mathfrak{I}}^{<\xi}$ durch $P_{\mathfrak{\Re}}^{<f(\xi)}$ ersetzt wird. Offenbar gilt stets $\mathrm{On}\left(T_{\alpha}(s)\right) \subseteq \mathrm{On}(s) \cup\{\alpha\}$.

Wir zeigen nun durch Induktion nach der Länge von $s$ :

$$
T_{\beta}(\hat{f}(s))=\hat{f}\left(T_{\alpha}(s)\right) \quad \text { und somit } \quad \hat{f}(s) \in \operatorname{dom}\left(T_{\beta}\right) .
$$

Abkürzung. $s^{\prime}:=\hat{f}(s), C^{\prime}:=\hat{f}(C)$.

$$
s=(): T_{\beta}\left(s^{\prime}\right)=\varphi_{-}^{\beta}=\left(\varphi_{-}^{\alpha}\right)^{\prime}=T_{\alpha}(s)^{\prime} .
$$

Sei nun $s=\left(x_{0}, \ldots, x_{n}\right)$ und $s_{0}=\left(x_{0}, \ldots, x_{n-1}\right)$.

$$
T_{\alpha}\left(s_{0}\right) \in \text { FOR kein Axiom, } n=2 m, x_{n}=*_{0} \text { : }
$$

Nach I.V. gilt $T_{\beta}\left(s_{0}^{\prime}\right)=T_{\alpha}\left(s_{0}\right)^{\prime}$, also $T_{\beta}\left(s_{0}^{\prime}\right) \in$ FOR kein Axiom und folglich: $T_{\beta}\left(s^{\prime}\right)$ $=\neg G_{m} \vee T_{\beta}\left(s_{0}^{\prime}\right)=\neg G_{m} \vee T_{\alpha}\left(s_{0}\right)^{\prime}=T_{\alpha}(s)^{\prime}$.

(T3) $T_{\alpha}\left(s_{0}\right)=C \in$ FOR kein Axiom und $n$ ungerade:

Nach I.V. gilt $T_{\beta}\left(\mathrm{s}_{0}^{\prime}\right)=C^{\prime}$.

$$
C=\Gamma\left(A_{0} \wedge A_{1}\right) \quad \text { und } \quad x_{n}=*_{i} \text { : }
$$

Dann $T_{\beta}\left(s_{0}^{\prime}\right)=\Gamma^{\prime}\left(A_{0}^{\prime} \wedge A_{1}^{\prime}\right)$ und somit $T_{\beta}\left(s^{\prime}\right)=\Gamma^{\prime}\left(A_{i}^{\prime}\right)=\Gamma\left(A_{i}\right)^{\prime}=T_{\alpha}(s)^{\prime}$

(T3.2) analog zu (T3.1).

(T3.3) $C=\Gamma(\exists x A(x))$ und $x_{n}=*_{0}$ :

Dann $T_{\alpha}(s)^{\prime}=\exists x A^{\prime}(x) \vee \Gamma^{\prime}\left(A^{\prime}\left(t_{k_{0}}\right)\right.$ ) (für ein gewisses $\left.k_{0} \in \mathbb{N}\right)$ und $T_{\beta}\left(s_{0}^{\prime}\right)=\Gamma^{\prime}\left(\exists x A^{\prime}(x)\right.$ ). Aus letzterem folgt $T_{\beta}\left(s^{\prime}\right)=\exists x A^{\prime}(x) \vee \Gamma^{\prime}\left(A^{\prime}\left(t_{k_{1}}\right)\right)$ (für ein gewisses $\left.k_{1} \in \mathbb{N}\right)$. Nach I.V. gilt außerdem für $i=0, \ldots, n$ und $k \in \mathbb{N}$ :

$$
A\left(t_{k}\right) \in \operatorname{Pos}\left(T_{\alpha}\left(\left(x_{0}, \ldots, x_{i-1}\right)\right)\right) \Leftrightarrow A^{\prime}\left(t_{k}\right) \in \operatorname{Pos}\left(T_{\beta}\left(\left(x_{0}, \ldots, x_{i-1}\right)^{\prime}\right)\right) .
$$

Daraus folgt $k_{0}=k_{1}$ und somit $T_{\beta}\left(s^{\prime}\right)=T_{\alpha}(s)^{\prime}$.

$$
C=\Gamma\left(t \in P_{\mathfrak{I}}\right) \text { und } x_{n}=*_{0} \text { : }
$$

Dann $T_{\beta}\left(s_{0}^{\prime}\right)=\Gamma^{\prime}\left(t \in P_{\mathscr{V}}\right)$ und folglich $T_{\beta}\left(s^{\prime}\right)=\mathfrak{U}\left(P_{\mathfrak{N}}, t\right) \vee \Gamma^{\prime}()=T_{\alpha}(s)^{\prime}$.

$$
C=\Gamma\left(t \notin P_{\mathscr{\mathscr { I }}}^{<\delta}\right) \text { und } x_{n}=\xi<\delta \text { : }
$$

Dann gilt $\xi, \delta \in \operatorname{dom}(f)$ und $f(\xi)<f(\delta)$. Ferner haben wir

$$
T_{\beta}\left(s_{0}^{\prime}\right)=\Gamma^{\prime}\left(t \notin P_{\mathfrak{I}}^{<f(\delta)}\right) \quad \text { und } \quad s^{\prime}=s_{0}^{\prime} \frown(f(\xi)) \text {, }
$$


also

$$
T_{\beta}\left(s^{\prime}\right)=\neg \mathfrak{A}\left(P_{\mathfrak{N}}^{<f(\xi)}, t\right) \vee \Gamma^{\prime}()=\left(\neg \mathfrak{U}\left(P_{\mathfrak{Q}}^{<\xi}, t\right) \vee \Gamma()\right)^{\prime}=T_{\alpha}(s)^{\prime} .
$$

wobei $A$ eine (negierte) $L$-Primformel ist: Dann $T_{\beta}\left(s_{0}^{\prime}\right)=\Gamma^{\prime}(A)$ und folglich $T_{\beta}\left(s^{\prime}\right)$ $=A \vee \Gamma^{\prime}()=T_{\alpha}(s)^{\prime}$.

\section{Zur Rekursivität von $D$}

Definition. Für $m, n \in \mathbb{N}$ und $f \in I(m, n)$ sei $\ulcorner f\urcorner:=\langle f(0), \ldots, f(m-1), n\rangle$, wobei \langle\rangle$: \bigcup_{n \in \mathbb{N}} \mathbb{N}^{n} \rightarrow \mathbb{N}$ eine der üblichen Kodierungsfunktionen sei. Ein Dilator $D$ heißt rekursiv, falls gilt:

(i) $D(n) \in \mathbb{N}$, für alle $n \in \mathbb{N}$.

(ii) Es gibt eine rekursive Funktion $d: \mathbb{N} \rightarrow \mathbb{N}$, so daß für alle $m, n \in \mathbb{N}$ und alle $f \in I(m, n)$ gilt $\ulcorner D(f)\urcorner=d(\ulcorner f\urcorner)$.

Unter der Voraussetzung, daß die in der Definition von $T_{F}$ (in Abschn. 2) vorkommenden Abzählungen $G_{0}, G_{1}, \ldots, t_{0}, t_{1}, \ldots$ rekursiv sind, ergibt sich die Rekursivität des oben definierten Dilators $D$ (bei Verwendung der Churchschen These) wie folgt:

zu (i): Für $n \in \mathbb{N}$ ist $T_{n}$ ein endlich verzweigter fundierter Baum und deshalb endlich; also $D(n)=\left\|T_{n}\right\|<\omega$.

zu (ii): Sei $f \in I(m, n)$ gegeben. Zu berechnen sind die Zahlen $D(m), D(n)$ und $D(f)(i)$ für $i<D(m)$. Dies geschieht folgendermaßen: Ausgehend von der Definition von $T_{F}$ in Abschn. 2 konstruiert man die endlichen Bäume $T_{m}$, $T_{n}$ und ordnet dann ihre Knoten gemäß der Kleene-Brouwer-Ordnung. So erhält man Folgen $\left(s_{0}, \ldots, s_{\bar{m}}\right),\left(r_{0}, \ldots, r_{\bar{n}}\right)$ mit $s_{i}, r_{j} \in \omega-\mathrm{SEQ}$ und $D(m)=\bar{m}+1, D(n)=\bar{n}+1$. Für $i<D(m)$ gilt $D(f)(i)=$ das eindeutig bestimmte $k<D(n)$ mit $\hat{f}\left(s_{i}\right)=r_{k}$.

\section{Literatur}

1. Girard, J.Y.: A survey of $\Pi_{2}^{1}$-logic. In: Barwise, J., Kaplan, D., Keisler, H.J., Suppes, P., Troelstra, A.S. (eds.), Logic, methodology and philosophy of science. VI. pp. 89-107. Amsterdam: North-Holland 1982

2. Girard, J.Y.: $\Pi_{2}^{1}-\operatorname{logic}$, Part 1: Dilators. Ann. Math. Logic 21, 75-219 (1981)

3. Girard, J.Y.: Introduction to $\Pi_{2}^{1}$-logic. Synthese 62, 191-216 (1985)

4. Girard, J.Y., Normann, D.: Set recursion and $\Pi_{2}^{1}$-logic. Ann. Pure Appl. Logic 28, 255-286 (1985)

5. Jäger, G.: Countable admissible ordinals and dilators. Z. Math. Logik Grundlagen Math. 32, $451-456(1986)$

6. Jervell, H.: Introducing homogeneous trees. In: Proc. Herbrand Symposion, Logic Colloquium 1981, pp. 147-158. Amsterdam: North-Holland 1982

7. Ressayre, J.P.: Bounding generalized recursive functions of ordinals by effective functors; a complement to the Girard theorem. In: Proc. Herbrand Symposion, Logic Colloquium 1981, pp. 251-279. Amsterdam: North-Holland 1982

8. Van de Wiele, J.: Recursive dilators and generalized recursions. In: Proc. Herbrand Symposion, Logic Colloquium 1981, pp. 325-332. Amsterdam: North-Holland 1982

9. Päppinghaus, P.: Ptykes in Gödels $T$ und Verallgemeinerte Rekursion über Mengen und Ordinalzahlen. Habilitationsschrift, Hannover 1985

10. Schütte, K.: Proof theory. Berlin Heidelberg New York: Springer 1977

Eingegangen am 12. Februar 1987 